\title{
Stabilization of Heterogeneous Multiagent Systems via Harmonic Control
}

\author{
Xianzhu Liu $\mathbb{D}^{1,2}$ Zhijian Ji $\mathbb{D},^{1}$ and Ting Hou $\mathbb{D}^{3}$ \\ ${ }^{1}$ Institute of Complexity Science, College of Automation and Electrical Engineering, Qingdao University, Qingdao 266071, China \\ ${ }^{2}$ School of Mathematics and Statistics, Qingdao University, Qingdao 266071, China \\ ${ }^{3}$ College of Mathematics and Systems Science, Shandong University of Science and Technology, Qingdao 266590, China
}

Correspondence should be addressed to Zhijian Ji; jizhijian@pku.org.cn

Received 11 April 2018; Revised 25 July 2018; Accepted 8 August 2018; Published 30 September 2018

Academic Editor: Zidong Wang

Copyright @ 2018 Xianzhu Liu et al. This is an open access article distributed under the Creative Commons Attribution License, which permits unrestricted use, distribution, and reproduction in any medium, provided the original work is properly cited.

Stabilizing multiagent systems including unstable agents shows the advantage of cooperation. This paper addresses the problem of stabilization of heterogeneous multiagent systems. Under cycle graphs, a sufficient condition for the stabilization problem via harmonic control is provided and an algorithm of designing the interconnection gains is presented. Furthermore, a sufficient and necessary condition for stabilization problem via harmonic control under cycle graphs is first given when the graph contains less than 5 nodes.

\section{Introduction}

In recent years, multiagent systems have broad applications in science and engineering areas such as consensus [1-11], controllability [12-22], and optimal control [23-25]. Multiagent systems are concerned chiefly with structures of networks and local information feedback including selfstate feedback and neighbor-state feedback. Designing decentralized controller with local information to realize stability is a basic problem in large-scale systems and multiagent systems [26-38]. Kim et al. [27] proposed a problem of stabilizability for multiagent systems with single-integrator dynamics by using external control inputs. Guan et al. extended the study to multiagent systems with general linear dynamics [28]. It is noted that [27, 28] only consider the identical agents. But in practice, many multiagent systems have different subsystems. These systems are called heterogeneous multiagent systems. For example, agents of flocks or satellite clusters might have different dynamic equations from each other due to their different masses or different structures [31]. In [32], a sufficient algebraic condition was provided for stabilization of heterogeneous multiagent systems in the case of static topology. The basic requirement of the sufficient condition in [32] is that each agent is stable or can be stabilized through self-state feedback. In the case where there exists an unstable agent which cannot be stabilized via self-state feedback, how to use neighbor-state feedback to stabilize multiagent systems is an important issue. Designing the gains of neighbor-state feedback has been applied in the plague control of some power networks $[31,35]$. However, there are rarely interesting results on such problems until recent years. To this day, the results for stabilization of heterogeneous multiagent systems are limited to cycle topologies, and only sufficient conditions are obtained. Reference [30] presented a sufficient condition for the stabilization problem, in which designing interconnection gains is called harmonic control and the system studied is composed of two subsystems. In [31], Zhu provided a sufficient condition for the stabilization of heterogeneous multiagent systems under directed cycle graphs. It will be challenging to explore necessary and sufficient conditions for stabilization of heterogeneous multiagent systems. The main reason is that heterogeneousness and complicated interconnections make the problem tricky. Therefore, what conditions can stabilize heterogeneous multiagent systems via harmonic control is still an open problem. 
This paper studies the stabilization of heterogeneous multiagent systems under directed communication topologies. The graphs include not only cycles but also paths, stars, and trees. We extended the results of [31]. The contributions are twofold:

(i) A sufficient condition for the stabilization problem via harmonic control is provided under cycle graphs. This condition is more general than the sufficient condition given in [31]. Besides, an approach is introduced to design the interconnection gains

(ii) When the multiagent system contains less than 5 agents, a necessary and sufficient algebraic condition is presented for this stabilization problem under cycle graphs. To the best of our knowledge, it is the first time to provide necessary and sufficient condition for stabilization problem of heterogeneous multiagent systems via harmonic control under cycle graphs

The structure of this paper is as follows. Section 2 presents some preliminaries and formulates the stabilization problem of multiagent systems. Section 3 provides the main results. Two numerical examples are given in Section 4 to show the applicability of the obtained results. Finally, conclusion is summarized in Section 5.

\section{Preliminaries}

Throughout this paper, the set of integers $\{1,2, \ldots, k\}$ is denoted by $\mathbb{N}_{k}$. With vertices representing agents and edges indicating the interconnections between them, graph theory proves to be a natural framework for modeling and treatment of multiagent systems. We consider directed graph rather than undirected graph. A directed graph is denoted by $\mathscr{G}=(\mathscr{V}, \mathscr{E}, \mathscr{A})$, where $\mathscr{V}=\{1,2, \cdots, n\}$ and $\mathscr{E} \subseteq \mathscr{V} \times \mathscr{V}$ represent the vertex and edge set, respectively. An edge $e_{i j}=(j, i) \in \mathscr{G}$ is represented by an arrow tailed at the node $j$ and headed toward the node $i$, which means node $i$ can receive information from $j$. The set of neighbors of node $i$ is denoted by $\mathcal{N}_{i}=\left\{j \in \mathscr{V}: e_{i j}=(j, i) \in \mathscr{E}, j \neq i\right\}$. The indegree of a vertex $k$ is the number of edges with head $k$. And the outdegree of a vertex $k$ is the number of edges with tail $k$. If every possible edge exists, the graph is said to be complete. A path of length $N$ from 1 to $N$ +1 is an ordered set of distinct vertices $\{1,2, \cdots, N+1\}$ such that $(i, i+1) \in \mathscr{E}$ for all $i \in \mathbb{N}_{N}$. An $N$-cycle is a path except for which $1=N+1$, meaning the path rejoins itself. $\mathscr{A}=\left[\omega_{i j}\right] \in \mathbb{R}^{n \times n}$ is the adjacency matrix whose $(i, j)$ entry is 1 if $(j, i)$ is one of $\mathscr{G}$ 's edges and 0 otherwise. A tree graph $\mathscr{T}_{i}$ with root $i$ is a graph that for each node other than $i$, there exists one and only one path from $i$ to this node. A node is called a leaf if its outdegree is zero, and two nodes are said to be in different branches when there is no path from any one of them to the another. A graph $\mathscr{G}$ is said to contain a spanning tree if there exists a tree whose nodes are all those in $\mathscr{V}$ and edges in the tree are also in $\mathscr{E}$. A star graph is a kind of special tree graph whose root is a neighbor of all nodes rest.
Let us consider a group of $N$ linear agents with information flow among them described by graph $\mathscr{G}=(\mathscr{V}, \mathscr{E})$ with $N=|\mathscr{V}|$, whose linear dynamics is

$$
\dot{x}_{i}=A_{i} x_{i}+B_{i} u_{i}+F_{i} v_{i}, \quad i=1, \ldots, N,
$$

where $x_{i} \in \mathbb{R}^{n_{i}}$ is the state vector of the $i$ th agent, and $A_{i} \in$ $\mathbb{R}^{n_{i} \times n_{i}}, B_{i} \in \mathbb{R}^{n_{i} \times p_{i}}$, and $F_{i} \in \mathbb{R}^{n_{i} \times q_{i}}$ are real matrices. $u_{i}$ is the self-state feedback law described by

$$
u_{i}=H_{i} x_{i}, \quad i=1, \ldots, N
$$

where $H_{i} \in \mathbb{R}^{q_{i} \times n_{i}} . \quad v_{i}$ is the neighbor-state feedback law described by

$$
v_{i}=\sum_{j \in \mathcal{N}_{i}} K_{i j} x_{j}, \quad i=1, \ldots, N,
$$

where $K_{i j} \in \mathbb{R}^{p_{i} \times n_{j}}$. The closed loop system is $\dot{x}=A x$, where $x=\left[x_{1}^{\mathrm{T}} x_{2}^{\mathrm{T}} \cdots x_{N}^{\mathrm{T}}\right]^{\mathrm{T}}$,

$$
A=\left[\begin{array}{cccc}
A_{1}+B_{1} H_{1} & \omega_{12} F_{1} K_{12} & \cdots & \omega_{1 N} F_{1} K_{1 N} \\
\omega_{21} F_{2} K_{21} & A_{2}+B_{2} H_{2} & \cdots & \omega_{2 N} F_{2} K_{2 N} \\
\vdots & \vdots & \ddots & \vdots \\
\omega_{N 1} F_{N} K_{N 1} & \omega_{N 2} F_{N} K_{N 2} & \cdots & A_{N}+B_{N} H_{N}
\end{array}\right],
$$

where $\omega_{i j}$ is the entry of the adjacent matrix of the graph $\mathscr{G}$.

Definition 1 [30]. The stabilization of a multiagent system is said to be solvable if there exists feedback law (2) and (3) such that the closed loop system (4) is stable.

In order to investigate more deeply the influence of neighbor-state feedback on the stabilization problem, the heterogeneous multiagent system (1) without self-state feedback gains is simplified to

$$
\dot{x}_{i}=A_{i} x_{i}+F_{i} v_{i}, \quad i=1, \ldots, N .
$$

Lemma 1 (Routh-Hurwitz criterion) [39]. A necessary and sufficient condition for polynomial

$$
p(s)=s^{n}+p_{1} s^{n-1}+\cdots+p_{n-1} s+p_{n},
$$

to be stable is that the determinants $\Delta_{1}, \cdots, \Delta_{n}$ are all positive, where

$$
\Delta_{r}=\left|\begin{array}{ccccc}
p_{1} & 1 & 0 & \cdots & 0 \\
p_{3} & p_{2} & p_{1} & \cdots & 0 \\
p_{5} & p_{4} & p_{3} & \cdots & 0 \\
\vdots & \vdots & \vdots & \ddots & \vdots \\
p_{2 \mathrm{r}-1} & p_{2 r-2} & p_{2 r-3} & \cdots & p_{r}
\end{array}\right|,
$$




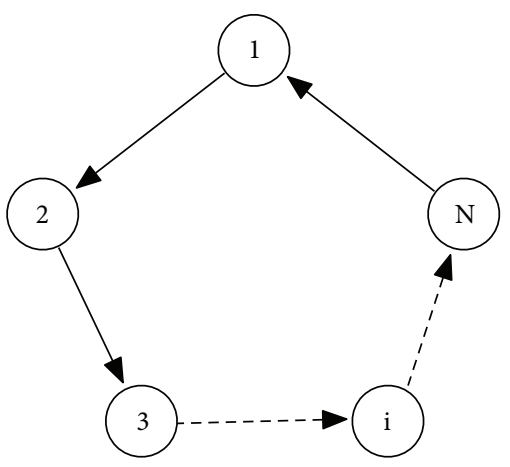

Figure 1: A cycle graph.

it is being understood that in each determinant, all the $p_{i}$ with subscripts that are either negative or greater than $n$ are to be replaced by zero. $\Delta_{r}(r=1, \cdots, n)$ is named as the Hurwitz determinant.

\section{Main Results}

In the section, we mainly investigate how to use neighborstate feedback to solve the stabilization problem when the multiagent systems are still unstable after using their selfstate feedback.

3.1. The Case without Self-State Feedback. Directed paths and directed cycles are basic ingredients for the investigation of stabilization of directed graphs. The analysis on path and cycle graphs is expected to provide insights for that of more complex structures. According to Appendix, for path graphs, star graphs, and tree graphs, the stabilizability is not affected by the neighbor-state feedback. That is to say, for path graphs, star graphs, or tree graphs, the stabilization problem is solvable if and only if each individual is stable or can be stabilized by its self-state feedback. Now, we consider the directed cycle graphs. The following assumption will be taken into account for the cycle graphs.

Assumption 1. $\left(A_{i}, F_{i}\right)$ is controllable, where $F_{i} \in \mathbb{R}^{n_{i} \times 1}$.

For the cycle graph $\mathscr{C}_{N}$ which is shown in Figure 1, the system matrix of the closed loop is

$$
A=\left[\begin{array}{ccccc}
A_{1} & 0 & \cdots & 0 & A_{1 N} \\
A_{21} & A_{2} & & & \\
& A_{32} & \ddots & & \\
& & \ddots & A_{N-1} & \\
& & & A_{N(N-1)} & A_{N}
\end{array}\right],
$$

where $A_{1 N}=F_{1} K_{1 N}, A_{i(i-1)}=F_{i} K_{i(i-1)}, i=1, \ldots, N-1$.
For convenience, we address the definition of harmonic polynomial of a diagonal block matrix $M=\operatorname{diag}$ $\left(M_{1}, \cdots, M_{N}\right)$.

Definition 2. For a diagonal block matrix $M=\operatorname{diag}\left(M_{1}, \cdots\right.$, $\left.M_{N}\right)$, if its characteristic polynomial is $\alpha(s):=\sum_{j=0}^{n} a_{j} s^{n-j}$, where $a_{0}=1$, then the harmonic polynomial of $M$ is

$$
h(s):=\sum_{j=0}^{N-1} a_{j} s^{N-1-j},
$$

where $a_{j}$ is the corresponding coefficients of the polynomial $\alpha(s)$.

Theorem 1. Consider the heterogeneous multiagent system (5) depicted by a cycle graph shown in Figure 1 with Assumption 1. The stabilization problem via harmonic control is solvable if the harmonic polynomial of $\operatorname{diag}\left(A_{1}, \cdots, A_{N}\right)$ is stable.

Proof 1. Since $\left(A_{i}, F_{i}\right)$ is controllable, it is assumed, without loss of generality, that $\left(A_{i}, F_{i}\right)$ is in the controllable canonical form.

$$
\begin{aligned}
A_{i} & =\left[\begin{array}{cccc}
0 & 1 & & \\
& & \ddots & \\
& & & 1 \\
-a_{i n_{i}} & -a_{i\left(n_{i}-1\right)} & \cdots & -a_{i 1}
\end{array}\right], \\
F_{i} & =\left[\begin{array}{c}
0 \\
\vdots \\
0 \\
1
\end{array}\right] .
\end{aligned}
$$

Design the general decentralized controllers as

$$
\begin{aligned}
v_{1} & =K_{1 N} x_{N}=\left[\begin{array}{llll}
k_{N n_{N}} & k_{N\left(n_{N}-1\right)} & \cdots & k_{N 1}
\end{array}\right] x_{N}, \\
v_{i+1} & =K_{(i+1) i} x_{i}=\left[\begin{array}{llll}
k_{i n_{i}} & k_{i\left(n_{i}-1\right)} & \cdots & k_{i 1}
\end{array}\right], \quad i=1, \ldots, N-1 .
\end{aligned}
$$

Then, we obtain the closed loop system as $\dot{x}=A x$, where $A$ is of the form as (8), where

$$
\begin{aligned}
A_{1 N} & =\left[\begin{array}{cccc}
0 & 0 & \cdots & 0 \\
k_{N n_{N}} & k_{N\left(n_{N}-1\right)} & \cdots & k_{N 1}
\end{array}\right]_{n_{1} \times n_{N}}, \\
A_{(i+1) i} & =\left[\begin{array}{cccc}
0 & 0 & \cdots & 0 \\
k_{i n_{i}} & k_{i\left(n_{i}-1\right)} & \cdots & k_{i 1}
\end{array}\right]_{n_{(i+1)} \times n_{i}}, \quad i=1, \ldots, N-1 .
\end{aligned}
$$


The characteristic polynomial of $A$ is $\gamma(s)=\operatorname{det}\left(s I_{n}\right.$ $-A)$. After a series of column-addition transformations, $\gamma(s)$ can be written as

$$
\gamma(s)=\operatorname{det}\left[\begin{array}{ccccc}
\widehat{A}_{1} & 0 & \cdots & 0 & \widehat{A}_{1 N} \\
\widehat{A}_{21} & \widehat{A}_{2} & & & \\
& \widehat{A}_{32} & \ddots & & \\
& & \ddots & \widehat{A}_{N-1} & \\
& & & \widehat{A}_{N(N-1)} & \widehat{A}_{N}
\end{array}\right] \text {, }
$$

where

$$
\begin{aligned}
\widehat{A}_{i} & =\left[\begin{array}{cccc}
0 & -1 & & \\
& \ddots & \ddots & \\
& & 0 & -1 \\
\alpha_{i}(s) & * & \cdots & *
\end{array}\right], \\
\widehat{A}_{r i} & =\left[\begin{array}{cccc}
0 & 0 & \cdots & 0 \\
\vdots & \vdots & \ddots & \vdots \\
-\sum_{j=1}^{n_{i}} k_{i j} s^{n_{i}-j} & * & \ldots & *
\end{array}\right] .
\end{aligned}
$$

$\alpha_{i}(s)$ is the characteristic polynomial of matrix $A_{i}$, and $*$ stands for the number of no interest. $r=1$ when $i=N$, otherwise $r=i+1$. Denote $\sum_{j=1}^{n_{i}} k_{i j} s^{n_{i}-j}$ by $\theta_{i}(s)$. Using Laplace expansion along the rows including -1 , then

$$
\begin{aligned}
& \gamma(s)=\operatorname{det}\left[\begin{array}{cccc}
\alpha_{1}(s) & 0 & \cdots & -\theta_{N}(s) \\
-\theta_{1}(s) & \alpha_{2}(s) & & \\
& \ddots & \ddots & \\
& & -\theta_{N-1}(s) & \alpha_{N}(s)
\end{array}\right] \\
& =\prod_{i=1}^{N} \alpha_{i}(s)-\prod_{i=1}^{N} \theta_{i}(s) .
\end{aligned}
$$

$$
\begin{aligned}
& \text { Let } \theta(s):=\sum_{i=0}^{n-N} b_{i} s^{(n-N)-i}:=\prod_{i=1}^{N} \theta_{i}(s) . \text { Then, } \\
& \begin{aligned}
\gamma(s)= & \alpha(s)-\theta(s)=s^{n}+a_{1} s^{n-1}+\cdots+a_{N-1} s^{n-N+1} \\
& +\left(a_{N}-b_{0}\right) s^{n-N}+\cdots+\left(a_{n}-b_{n-N}\right) .
\end{aligned}
\end{aligned}
$$

Since $b_{i}(i=0, \cdots, n-N)$ is not fixed, if $\alpha(s)$ is stable, there exist appropriate values of $b_{i}$ s which can make $\gamma(s)$ stable. For example, one can take $n-N+1$ small enough positive real numbers $\lambda_{j}=\varepsilon$, where $j=1, \ldots,(n-N+1)$ and $\varepsilon>0$. Let

$$
\begin{aligned}
p(s):= & \prod_{j=1}^{n-N+1}\left(s+\lambda_{j}\right)=(s+\varepsilon)^{n-N+1}=s^{n-N+1} \\
& +p_{1} s^{n-N}+\cdots+p_{n-N} s+p_{n-N+1} .
\end{aligned}
$$

Using polynomial long division, the result of dividing $\alpha(s)$ by $p(s)$ is quotient $g(s)=s^{N-1}+g_{1} s^{N-2}+\cdots+g_{N-2} s+$ $g_{N-1}$ and remainder $\theta(s)$. Since $p_{i}=C_{n-N+1}^{i} \varepsilon^{i}$, it is obvious that $p_{i} \rightarrow 0$ as $\varepsilon \rightarrow 0$. Consequently, $g_{i} \rightarrow a_{i}$ as $\varepsilon \rightarrow 0$, where $i=1, \ldots, N-1$. That is to say, $g(s) \rightarrow h(s)$ as $\varepsilon \rightarrow 0$. It follows that if $h(s)$ is stable, then $g(s)$ is stable. Let the characteristic polynomial $\gamma(s)$ be $p(s) * g(s)$, accordingly $\gamma(s)$ is stable. Using polynomial factorization, $K_{i j}$ can be assigned by the coefficients of the factors of $\theta(s)$.

In the following, we propose an algorithm to obtain $K_{i j}$ in which case $\left(A_{i}, F_{i}\right)$ is in the controllable standard form. The algorithm is described as follows:

Step 1. If $\left(A_{i}, F_{i}\right)$ is controllable, then calculate the characteristic polynomial $\alpha_{i}(s)$ of $A_{i}$.

Step 2. Get the polynomial $\alpha(s)=\prod_{i=1}^{N} \alpha_{i}(s)$.

Step 3. Take $n-N+1$ small enough positive real numbers $\varepsilon_{i}>0$ to obtain the polynomial $p(s)=\prod_{i=1}^{n-N+1}\left(s+\varepsilon_{i}\right)$.

Step 4. Divide $\alpha(s)$ by $p(s)$ to obtain the remainder $\theta(s)$.

Step 5. Factorize the polynomial $\theta(s)$ and $K_{i j}$ can be assigned by the coefficients of the factors of $\theta(s)$.

Theorem 1 provides a sufficient condition for the stabilization via harmonic control under cycle graphs. To the best of our knowledge, sufficient and necessary conditions for stabilization via harmonic control under cycle graphs have not yet been studied in detail. Due to the difficulty of the problem, we only provide sufficient and necessary conditions for the stabilization via harmonic control under cycle graphs with less than 5 nodes.

Lemma 2. If the polynomial (6) is stable, then the polynomial

$$
q(s)=s^{m}+p_{1} s^{m-1}+\cdots+p_{m-1} s+p_{m}
$$

is stable, where $m \in\{1,2,3\}$ and $n \geq m$.

Proof 2 .

(i) If $m=1$, then $q(s)=s+p_{1}$. Equation (6) is stable; it follows that $p_{1}>0$. Hence, $q(s)$ is stable.

(ii) If $m=2$, then $q(s)=s^{2}+p_{1} s+p_{2}$. Since (6) is stable, it follows that $p_{i}>0, i=1,2$. Thus, $p_{1}>0, p_{1} p_{2}>0$. By Routh-Hurwitz criterion, $q(s)$ is stable.

(iii) If $m=3$, then $q(s)=s^{3}+p_{1} s^{2}+p_{2} s+p_{3}$. Since (6) is stable, it follows that $p_{i}>0, i=1,2,3$, and $\Delta_{1}^{p}=$ $p_{1}>0, \Delta_{2}^{p}>0$. For $q(s), \Delta_{1}^{q}=p_{1}>0, \Delta_{2}^{q}=\Delta_{2}^{p}>0$, and 


$$
\Delta_{3}^{q}=\operatorname{det}\left[\begin{array}{ccc}
p_{1} & 1 & 0 \\
p_{3} & p_{2} & p_{1} \\
0 & 0 & p_{3}
\end{array}\right]=p_{3} \Delta_{2}^{q}>0 .
$$

Hence, $q(s)$ is stable.

Theorem 2. Consider the heterogeneous multiagent system (5), $N \in\{2,3,4\}$, depicted by a cycle graph with Assumption 1. The stabilization problem via harmonic control is solvable if and only if the harmonic polynomial of $\operatorname{diag}\left(A_{1}, \cdots, A_{N}\right)$ is stable.

Proof 3. According to Theorem 1, the sufficiency is obvious. So, we only need to prove the necessity.

(Necessity) Suppose that $N \in\{2,3,4\}$. According to Lemma 2, if $\alpha(s)$ is stable, then $s^{N-1}+a_{1} s^{N-2}+\cdots+a_{N-1}$ is stable.

Corollary 1. If $N=2$, then the stabilization problem via harmonic control is solvable if and only if $\operatorname{tr}\left(A_{1}\right)+\operatorname{tr}\left(A_{2}\right)<0$, where $\operatorname{tr}(A)$ denotes the trace of matrix $A$.

Proof 4. (Necessity) According to Theorem 2, if the system composed of two agents can be stabilized, then $a_{1}=a_{11}+$ $a_{21}>0$. Since $a_{11}=-\operatorname{tr}\left(A_{1}\right)$ and $a_{21}=-\operatorname{tr}\left(A_{2}\right)$, it follows that $\operatorname{tr}\left(A_{1}\right)+\operatorname{tr}\left(A_{2}\right)<0$.

(Sufficiency) If $\operatorname{tr}\left(A_{1}\right)+\operatorname{tr}\left(A_{2}\right)<0$, then $a_{1}=a_{11}+a_{21}=$ $\left(-\operatorname{tr}\left(A_{1}\right)-\operatorname{tr}\left(A_{2}\right)\right)>0$. According to Theorem 1, for the system composed of two agents, if $h(s)=s+a_{1}$ is stable, i.e., $a_{1}>0$, then the system can be stabilized via harmonic control.

Remark 1. When $N=2$, if $\operatorname{tr}\left(A_{1}\right)+\operatorname{tr}\left(A_{2}\right)<0$, according to Corollary 1, we can choose $\lambda_{j}<0(j=1,2 \cdots, n)$ such that $\sum_{j}^{n} \lambda_{j}=\left(\operatorname{tr}\left(A_{1}\right)+\operatorname{tr}\left(A_{2}\right)\right)$. Then, the characteristic polynomial $\gamma(s)=\prod_{j=1}^{n}\left(s-\lambda_{j}\right)$. Consequently, $\theta(s)=\alpha(s)-\gamma(s)$. Using polynomial factorization, $K_{i j}$ can be assigned by the coefficients of the factors of $\theta(s)$.

Remark 2. It is challenging to obtain the sufficient and necessary condition for the stabilization problem via harmonic control under cycle graph, although we solved the stabilization problem where $N \in\{2,3,4\}$. If $N>4$, then the sufficient and necessary conditions will be difficult to obtain. For example, suppose that $N=5$ and the characteristic polynomial of $A$ is $\alpha(s)=s^{5}+s^{4}+3 s^{3}+2.3 s^{2}+2 s+1.2 . \alpha(s)$ is stable. However, the harmonic polynomial $h(s)=s^{4}+s^{3}+3 s^{2}+2.3 s+2$ is not stable. Consequently, when $N>4$, it is difficult to explore the necessary condition for the stabilization problem via harmonic control under cycle graph.

3.2. The Case with Self-State Feedback. In case the graph with self-state feedback does not contain cycles, the above discussion implies that the stabilization problem is solvable if each pair $A_{i}+B_{i} H_{i}$ is stabilizable. For the cycle graph, even if the pair $A_{i}+B_{i} H_{i}$ is unstable, the stabilization problem is solvable as long as the harmonic polynomial of the matrix $\operatorname{diag}\left(A_{1}+B_{1} H_{1}, \cdots, A_{N}+B_{N} H_{N}\right)$ is stable.

\section{Examples}

Example 1. Given a system with two agents whose state matrices are as follows:

$$
\begin{aligned}
& A_{1}=\left[\begin{array}{lll}
0 & 1 & 0 \\
0 & 0 & 1 \\
-9 & 1 & -3
\end{array}\right], \\
& F_{1}=\left[\begin{array}{l}
0 \\
0 \\
1
\end{array}\right], \\
& A_{2}=\left[\begin{array}{ll}
0 & 1 \\
-9 & 1
\end{array}\right], \\
& F_{2}=\left[\begin{array}{l}
0 \\
1
\end{array}\right] .
\end{aligned}
$$

Clearly, every agent is unstable. $\left(A_{i}, F_{i}\right)\left(i \in \mathbb{N}_{2}\right)$ is controllable, and $\operatorname{tr}\left(A_{1}\right)+\operatorname{tr}\left(A_{2}\right)=-2<0$. According to Corollary 1 , the system can be stabilized by constructing $K_{12}$ and $K_{21}$. Assume $\lambda_{i}<0\left(i \in \mathbb{N}_{5}\right)$ is the eigenvalue of $A$, it only needs to satisfy that $\sum_{i=1}^{5} \lambda_{i}=-2$, then the overall system can be stabilized. So, we can take $\lambda_{1}=-0.2, \lambda_{2}=-0.3, \lambda_{3}=$ $-0.4, \lambda_{4}=-0.5, \lambda_{5}=-0.6$, and then

$$
\begin{aligned}
\gamma(s) & =\left(s-\lambda_{1}\right)\left(s-\lambda_{2}\right)\left(s-\lambda_{3}\right)\left(s-\lambda_{4}\right)\left(s-\lambda_{5}\right) \\
& =s^{5}+2 s^{4}+1.55 s^{3}+0.58 s^{2}+0.1044 s+0.0072 .
\end{aligned}
$$

Further,

$$
\theta(s)=\alpha(s)-\gamma(s)=3.45 s^{3}+36.42 s^{2}-18.1044 s+80.9928
$$

Assume that $K_{21}=\left[\begin{array}{lll}k_{13} & k_{12} & k_{11}\end{array}\right]$ and $K_{12}=\left[\begin{array}{ll}k_{22} & 1\end{array}\right]$, then one can get $k_{22}=11.2114$, and $K_{21}=$ $\left[\begin{array}{lll}7.2242 & -2.2592 & 3.45\end{array}\right]$.

The decentralized controller can be designed as

$$
\begin{aligned}
& v_{1}=\left[\begin{array}{ll}
11.2114 & 1
\end{array}\right] x_{2}, \\
& v_{2}=\left[\begin{array}{lll}
7.2242 & -2.2592 & 3.45
\end{array}\right] x_{1} .
\end{aligned}
$$

Figure 2 shows the asymptotical stability of the closed loop system. 


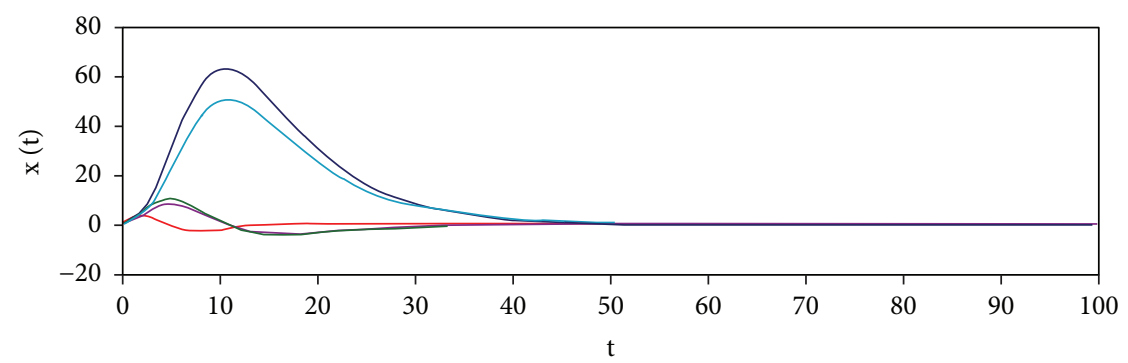

FIGURE 2: Stabilization via harmonic control.

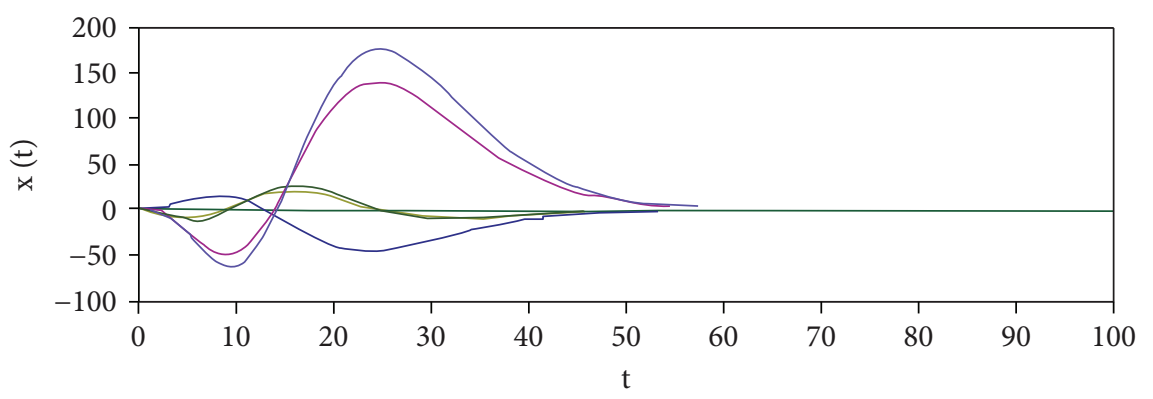

FIGURE 3: Stabilization via harmonic control.

Example 2. Consider the heterogeneous multiagent system composed of three agents, and

$$
\begin{aligned}
A_{1}= & {\left[\begin{array}{llll}
0 & 1 & 0 & 0 \\
0 & 0 & 1 & 0 \\
0 & 0 & 0 & 1 \\
5 & -3 & -6 & -8
\end{array}\right], } \\
A_{2} & =\left[\begin{array}{lll}
0 & 1 & 0 \\
0 & 0 & 1 \\
9 & 2 & -5
\end{array}\right], \\
A_{3} & =\left[\begin{array}{ll}
0 & 1 \\
-3 & 1
\end{array}\right], \\
F_{1}^{\mathrm{T}} & =\left[\begin{array}{lll}
0 & 0 & 0
\end{array}\right], \\
F_{2}^{\mathrm{T}} & =\left[\begin{array}{lll}
0 & 0 & 1
\end{array}\right], \\
F_{3}^{\mathrm{T}} & =\left[\begin{array}{lll}
0 & 1
\end{array}\right] .
\end{aligned}
$$

Apparently, every agent is unstable. According to Theorem 2 of [31], $f(s)=s^{7}+12 s^{6}+34 s^{5}+3 s^{4}-50 s^{3}+13 s^{2}-154 s-$ 193 is unstable. In this case, Theorem 2 of [31] becomes invalid. But the harmonic polynomial, $h(s)=s^{2}+12 s+34$, is stable; according to Theorem 1, the system can be stabilized via harmonic control. Taking $\varepsilon=0.3$, then the decentralized controller can be designed as

$$
\begin{aligned}
& v_{1}=\left[\begin{array}{llll}
1.3148 & 1
\end{array}\right] x_{3}, \\
& v_{2}=\left[\begin{array}{llll}
27.3819 & -21.744 & -30.6617 & -40.428
\end{array}\right] x_{1}, \\
& v_{3}=\left[\begin{array}{lll}
3.7497 & -2.5424 & 1
\end{array}\right] x_{2} .
\end{aligned}
$$

Figure 3 shows the asymptotical stability of the closed loop system.

\section{Conclusion}

In this paper, we have demonstrated the problem of stabilization of heterogeneous multiagent systems via harmonic control. The framework puts emphasis on the ability of systems to stabilize themselves when each agent only uses its neighbors' state information as feedback. For the overall interconnected system including unstable agents, we have presented that its stabilizability is affected by the structure of the interconnection topology and the interaction of its agents. Under cycle graphs, a sufficient condition and the design of harmonic control interconnection gains are provided. When the graph contains less than 5 nodes, the sufficient and necessary condition for stabilization problem via harmonic control under cycle graph can be given. In future work, the general graphs and the sufficient and necessary condition for stabilization via harmonic control will be considered. 

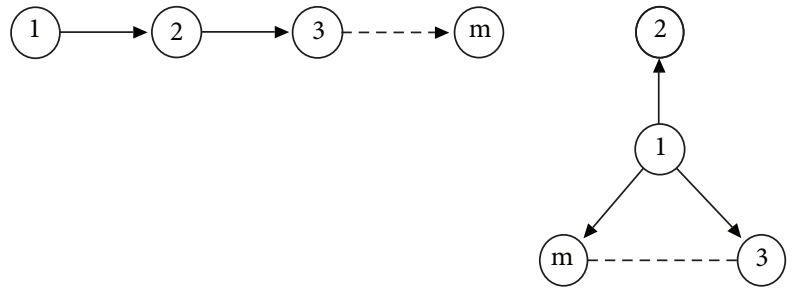

(a)

(b)

FIgURE 4: A path graph and a star graph.

\section{Appendix}

\section{Stabilization without Self-State Feedback of Path, Star and Tree Graphs}

Proposition A.1. For a path or star graph, the stabilization problem only via neighbor-state feedback is solvable if and only if each agent is stable.

Proof 5. For a path graph, which is showed in Figure 4(a), the system matrix of the closed loop is

$$
A=\left[\begin{array}{cccc}
A_{1} & & & \\
F_{2} K_{21} & A_{2} & & \\
& \ddots & \ddots & \\
& & F_{m} K_{m(m-1)} & A_{m}
\end{array}\right] .
$$

Therefore, the characteristic polynomial of $A$ is $\alpha(s)=$ $\operatorname{det}\left(s I_{n}-A\right)=\prod_{i=1}^{m} \alpha_{i}(s)$.

Obviously, the roots of $\alpha(s)$ cannot be changed only by neighbor-state feedback. If the path graph has an unstable agent $i$, the roots of $A_{i}$ are also the roots of $\alpha(s)$. Consequently, the stabilization problem only via neighbor-state feedback is solvable if and only if each agent is stable.

For a star graph, which is showed in Figure 4(b), the system matrix of the closed loop is

$$
A=\left[\begin{array}{cccc}
A_{1} & & & \\
F_{2} K_{21} & A_{2} & & \\
\vdots & & \ddots & \\
F_{m} K_{m 1} & & & A_{m}
\end{array}\right] .
$$

Similar to the proof of the path graph, if the star graph has an unstable agent, the stabilization problem only via neighbor-state feedback is solvable if and only if each agent is stable.

Proposition A.2. If a graph has at least one unstable node whose indegree or outdegree is equal to zero, then the stabilization problem only via neighbor-state feedback is unsolvable.

Proof 6. Without loss of generality, assume that the indegree of the first vertex is equal to zero and $A_{1}$ is not stable. Then, $A$ is in the form of

$$
A=\left[\begin{array}{cc}
A_{1} & 0 \\
\tilde{A}_{21} & \tilde{A}_{2}
\end{array}\right],
$$

where $A_{1} \in \mathbb{R}^{n_{1} \times n_{1}}$ and $\tilde{A}_{2} \in \mathbb{R}^{\left(n-n_{1}\right) \times\left(n-n_{1}\right)}$. Obviously, the roots of $A_{1}$ are also the roots of $\alpha(s)$. Consequently, the stabilization problem only via neighbor-state feedback is unsolvable.

The case of outdegree is equal to zero can be similarly obtained.

Proposition A.3. For a tree graph, the stabilization problem only via neighbor-state feedback is solvable if and only if each agent is stable.

Proof 7. If the root or one leaf is unstable, by Proposition A.2, the stabilization problem only via neighbor-state feedback is unsolvable.

Suppose that the root and all leaves are stable. Every time we remove a leaf from the tree, what remains is still a tree whose stabilizability is equivalent to the original one. So long as an unstable leaf appears after removing the leaves, the stabilization problem only via neighbor-state feedback is unsolvable by Proposition A.2. Thus, the proof is achieved.

\section{Data Availability}

The data used to support the findings of this study are included within the article.

\section{Conflicts of Interest}

The authors declare that they have no conflicts of interest.

\section{Acknowledgments}

This work was supported by the National Natural Science Foundation of China under Grant nos. 61873136, 61374062, 61603288, and 61673013, the Natural Science Foundation of Shandong Province for Distinguished Young Scholars under Grant no. JQ201419, and the Natural Science Foundation of Shandong Province under Grant no. ZR2016JL022. We would like to thank the referees of this paper for their perceptive and helpful comments.

\section{References}

[1] Y. Zheng and L. Wang, "Distributed consensus of heterogeneous multi-agent systems with fixed and switching topologies," International Journal of Control, vol. 85, no. 12, pp. 1967-1976, 2012.

[2] K. Liu, Z. Ji, G. Xie, and L. Wang, "Consensus for heterogeneous multi-agent systems under fixed and switching topologies," Journal of the Franklin Institute, vol. 352, no. 9, pp. 3670-3683, 2015. 
[3] J. Xi, M. He, H. Liu, and J. Zheng, "Admissible output consensualization control for singular multi-agent systems with time delays," Journal of the Franklin Institute, vol. 353, no. 16, pp. 4074-4090, 2016.

[4] N. Cai, C. Diao, and M. J. Khan, "A novel clustering method based on quasi-consensus motions of dynamical multiagent systems," Complexity, vol. 2017, Article ID 4978613, 8 pages, 2017.

[5] Y. Zheng and L. Wang, "Consensus of switched multi-agent systems," IEEE Transactions on Circuits and Systems II: Express Briefs, vol. 63, no. 3, pp. 314-318, 2016.

[6] F. Xiao and T. Chen, "Adaptive consensus in leader-following networks of heterogeneous linear systems," IEEE Transactions on Control of Network Systems, p. 1, 2017.

[7] L. Ma, Z. Wang, Q. L. Han, and Y. Liu, "Consensus control of stochastic multi-agent systems: a survey," Science China Information Sciences, vol. 60, no. 12, article 120201, 2017.

[8] L. Ma, Z. Wang, and H. K. Lam, "Event-triggered mean-square consensus control for time-varying stochastic multi-agent system with sensor saturations," IEEE Transactions on Automatic Control, vol. 62, no. 7, pp. 3524-3531, 2017.

[9] F. Xiao, T. Chen, and H. Gao, "Consensus in time-delayed multi-agent systems with quantized dwell times," Systems \& Control Letters, vol. 104, pp. 59-65, 2017.

[10] Y. Guan and L. Wang, "Structural controllability of multiagent systems with absolute protocol under fixed and switching topologies," Science China Information Sciences, vol. 60, no. 9, article 092203, 2017.

[11] J. Xi, Z. Fan, H. Liu, and T. Zheng, "Guaranteed-cost consensus for multi-agent networks with Lipschitz nonlinear dynamics and switching topologies," International Journal of Robust and Nonlinear Control, vol. 28, no. 7, pp. 28412852, 2018.

[12] D. Ding, Z. Wang, Q. L. Han, and G. Wei, "Neural-networkbased output-feedback control under round-Robin scheduling protocols," IEEE Transactions on Cybernetics, no. 99, pp. 1-13, 2018.

[13] D. Ding, Z. Wang, Q. L. Han, and G. Wei, "Security control for discrete-time stochastic nonlinear systems subject to deception attacks," IEEE Transactions on Systems, Man, and Cybernetics: Systems, vol. 48, no. 5, pp. 779-789, 2018.

[14] Z. Zuo, Q. L. Han, B. Ning, X. Ge, and X. M. Zhang, "An overview of recent advances in fixed-time cooperative control of multi-agent systems," IEEE Transactions on Industrial Informatics, vol. 14, no. 6, pp. 2322-2334, 2018.

[15] X. M. Zhang, Q. L. Han, and X. Yu, "Survey on recent advances in networked control systems," IEEE Transactions on Industrial Informatics, vol. 12, no. 5, pp. 1740-1752, 2016

[16] X. Ge, Q. L. Han, D. Ding, X. M. Zhang, and B. Ning, “A survey on recent advances in distributed sampled-data cooperative control of multi-agent systems," Neurocomputing, vol. 275, pp. 1684-1701, 2018.

[17] Z. Ji, Z. Wang, H. Lin, and Z. Wang, "Interconnection topologies for multi-agent coordination under leader-follower framework," Automatica, vol. 45, no. 12, pp. 2857-2863, 2009.

[18] X. Liu and Z. Ji, "Controllability of multi-agent systems based on path and cycle graphs," International Journal of Robust and Nonlinear Control, vol. 28, no. 1, pp. 296-309, 2018.

[19] Z. Ji and H. Yu, "A new perspective to graphical characterization of multi-agent controllability," IEEE Transactions on Cybernetics, vol. 47, no. 6, pp. 1471-1483, 2017.
[20] Y. Guan, Z. Ji, L. Zhang, and L. Wang, "Controllability of multi-agent systems under directed topology," International Journal of Robust and Nonlinear Control, vol. 27, no. 18, pp. 4333-4347, 2017.

[21] Y. Chao and Z. Ji, "Necessary and sufficient conditions for multi-agent controllability of path and star topologies by exploring the information of second-order neighbours," IMA Journal of Mathematical Control and Information, article dnw013, 2016.

[22] N. Cai, M. He, Q. Wu, and M. J. Khan, "On almost controllability of dynamical complex networks with noises," Journal of Systems Science and Complexity, 2017.

[23] Y. Yuan, H. Yuan, Z. Wang, L. Guo, and H. Yang, "Optimal control for networked control systems with disturbances: a delta operator approach," IET Control Theory \& Applications, vol. 11, no. 9, pp. 1325-1332, 2017.

[24] Y. Yuan, Z. Wang, P. Zhang, and H. Dong, "Nonfragile nearoptimal control of stochastic time-varying multiagent systems with control- and state-dependent noises," IEEE Transactions on Cybernetics, no. 99, pp. 1-13, 2018.

[25] Y. Yuan, Z. Wang, and L. Guo, "Event-triggered strategy design for discrete-time nonlinear quadratic games with disturbance compensations: the noncooperative case," IEEE Transactions on Systems, Man, and Cybernetics: Systems, no. 99, pp. 1-12, 2017.

[26] J. Xiong and J. Lam, "Stabilization of linear systems over networks with bounded packet loss," Automatica, vol. 43, no. 1, pp. 80-87, 2007.

[27] H. Kim, H. Shim, J. Back, and J. H. Seo, "Stabilizability of a group of single integrators and its application to decentralized formation problem," in 2011 50th IEEE Conference on Decision and Control and European Control Conference, pp. 4829-4834, Orlando, FL, USA, 2011.

[28] Y. Guan, Z. Ji, L. Zhang, and L. Wang, "Decentralized stabilizability of multi-agent systems under fixed and switching topologies," Systems \& Control Letters, vol. 62, no. 5, pp. 438-446, 2013.

[29] L. Zhang and E. K. Boukas, "Stability and stabilization of Markovian jump linear systems with partly unknown transition probabilities," Automatica, vol. 45, no. 2, pp. 463-468, 2009.

[30] Z. S. Duan, L. Huang, J. Z. Wang, and L. Wang, "Harmonic control between two systems," Acta Automatica Sinica, vol. 29, no. 1, pp. 14-22, 2003.

[31] J. Zhu, "Stabilization and synchronization for a heterogeneous multi-agent system via harmonic control," Systems \& Control Letters, vol. 66, pp. 1-7, 2014.

[32] M. Franceschelli, A. Gasparri, A. Giua, and G. Ulivi, "Decentralized stabilization of heterogeneous linear multi-agent systems," in 2010 IEEE International Conference on Robotics and Automation, pp. 3556-3561, Anchorage, AK, USA, 2010.

[33] S. Loizu, D. Dimarogonas, and K. Kyriakopoulos, "Decentralized feedback stabilization of multiple nonholonomic agents," in IEEE International Conference on Robotics and Automation, 2004. Proceedings. ICRA '04. 2004, vol. 3, pp. 3012-3017, New Orleans, LA, USA, 2004.

[34] J. C. Willems and M. Ikeda, "Decentralized stabilization of large-scale interconnected systems," in Analysis and Optimization of Systems, pp. 236-244, Springer, 2006.

[35] X. Liu and Y. Zou, "Harmonic control based on Hadamard product LMI approach," in 2008 10th International Conference 
on Control, Automation, Robotics and Vision, pp. 1401-1406, Hanoi, Vietnam, 2009.

[36] X. Liu, Q. Zhao, and S. Zhong, "Stability analysis of a class of switched nonlinear systems with delays: a trajectory-based comparison method," Automatica, vol. 91, pp. 36-42, 2018.

[37] Z. Duan and L. Huang, "Two kinds of harmonic problems in control systems," Journal of Systems Science and Complexity, vol. 22, no. 4, pp. 587-596, 2009.

[38] Y. Guan, Z. Ji, L. Zhang, and L. Wang, "Quadratic stabilisability of multi-agent systems under switching topologies," International Journal of Control, vol. 87, no. 12, pp. 2657-2668, 2014.

[39] G. James, Advanced Modern Engineering Mathematics, Pearson, Harlow, UK, 3rd edition, 2004. 


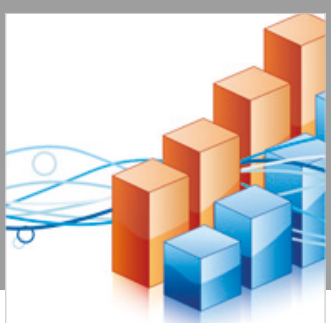

Advances in

Operations Research

\section{-n-m}
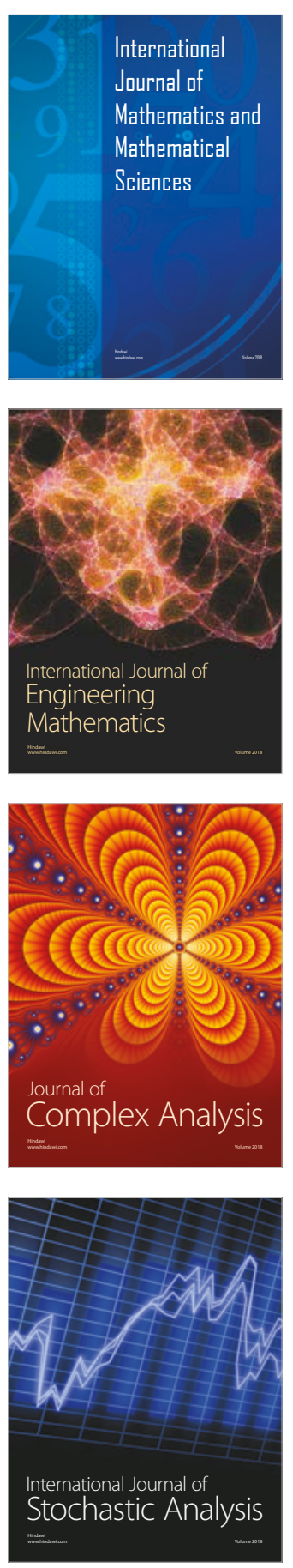
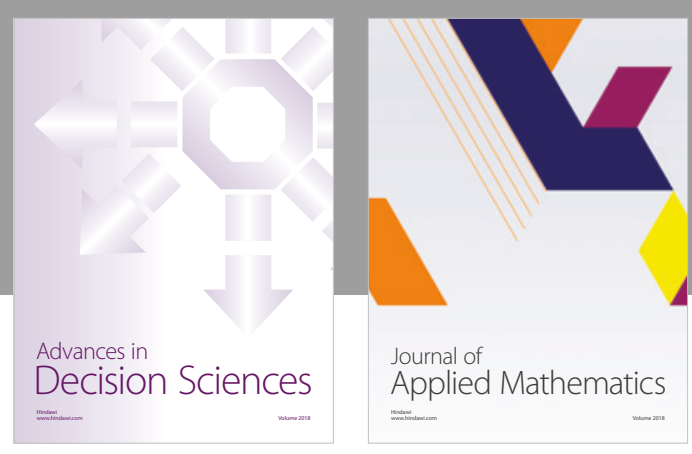

Journal of

Applied Mathematics
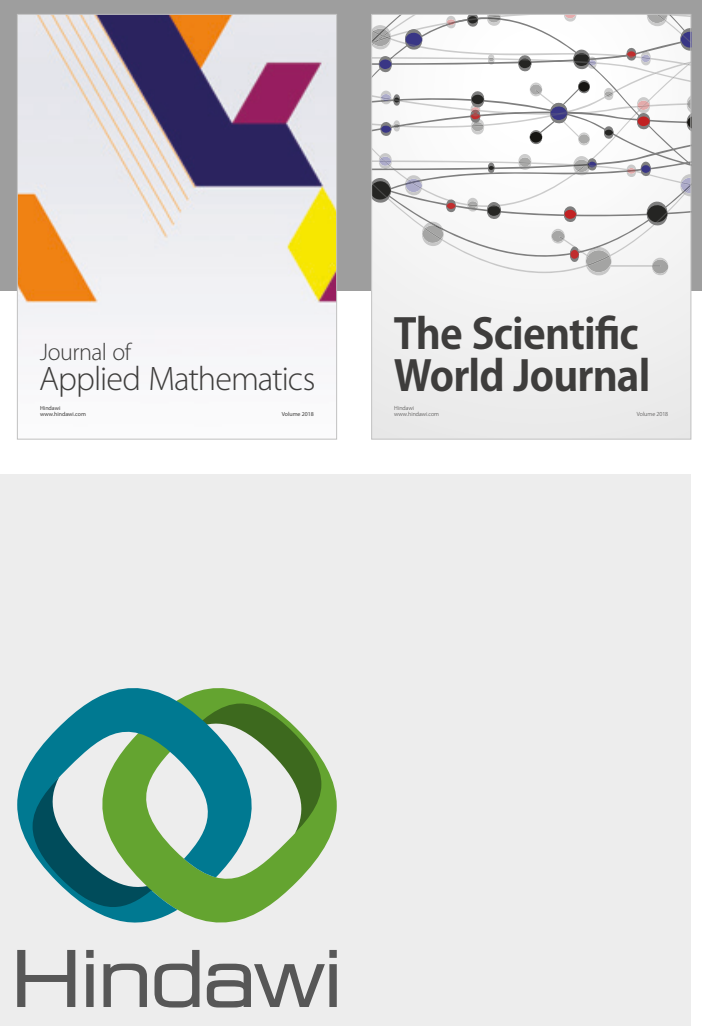

Submit your manuscripts at

www.hindawi.com

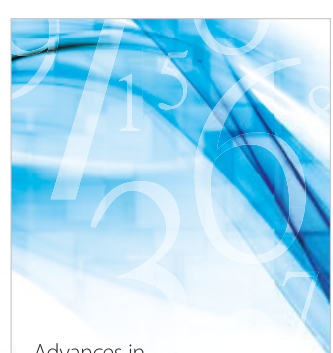

Advances in
Numerical Analysis
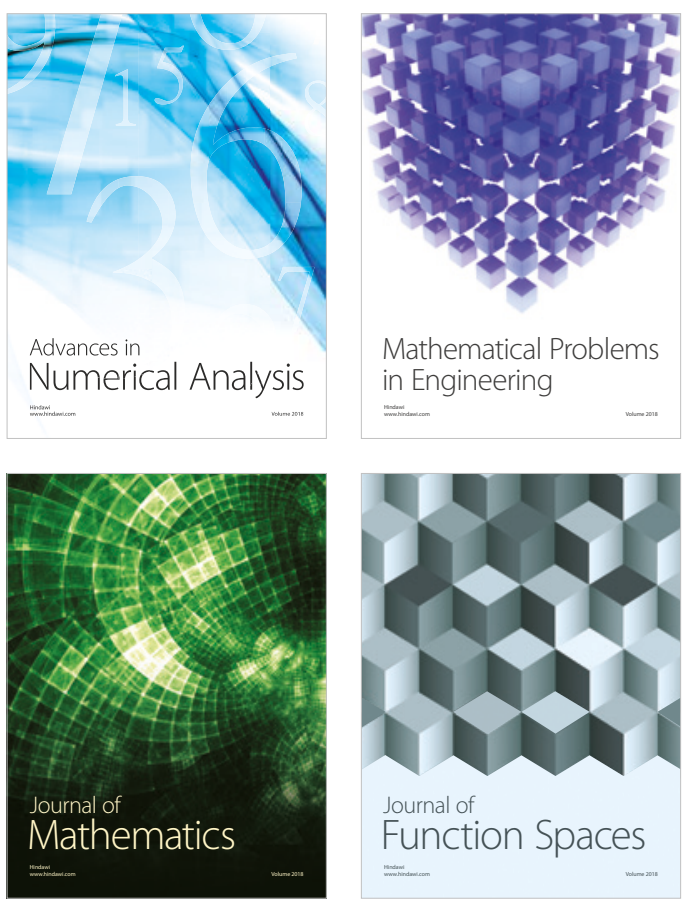

Mathematical Problems in Engineering

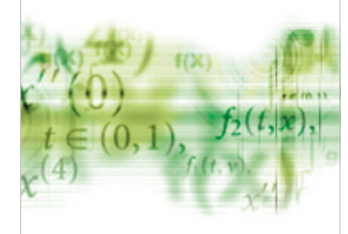

International Journal of

Differential Equations

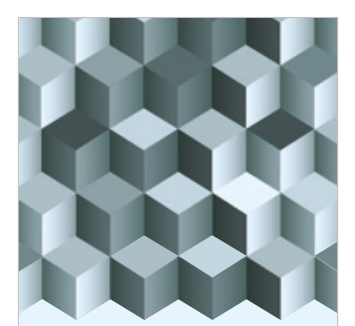

Journal of

Function Spaces
The Scientific

World Journal

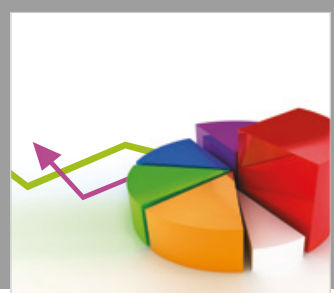

Journal of

Probability and Statistics
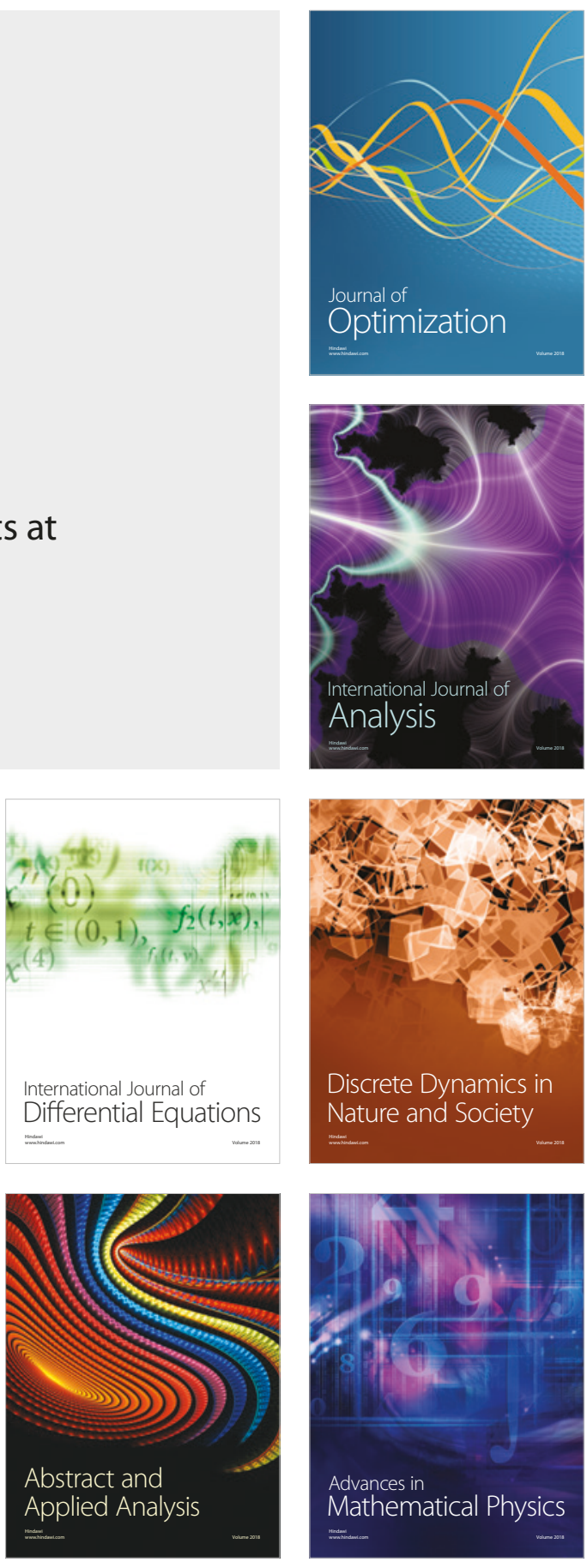